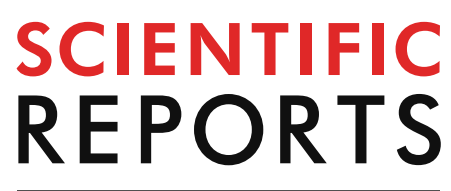

natureresearch

Check for updates

\title{
The importance of socioeconomic
} position in smoking, cessation and environmental tobacco smoke exposure during pregnancy

\author{
Joana Madureira ${ }^{1,2,4}$, Alexandra Camelo ${ }^{1,2,4}$, Ana Inês Silva ${ }^{1,2,3}$, Ana Teresa Reis ${ }^{1,2}$, \\ Filipa Esteves ${ }^{1,2}$, Ana Isabel Ribeiro ${ }^{2}$, João Paulo Teixeira ${ }^{1,2}$ \& Carla Costa ${ }^{1,2}$
}

Tobacco is still a leading cause of premature death and morbidity. Particular attention has been given to pregnant women due to the scientific evidence on the importance of early life exposures for disease onset later in life. The purpose of this study was to assess smoking prevalence, smoking cessation rate and environmental tobacco smoke (ETS) exposure, and the role of socioeconomic position (SEP) on these behaviors among pregnant women. Cross-sectional data of 619 pregnant women, aged between 18 and 46 years, from Porto Metropolitan Area, Portugal, on current smoking, ETS exposure and SEP indicators was collected, face-to-face, using a questionnaire filled in during a personal interview at the postpartum hospital stay. The smoking prevalence, and ETS exposure among nonsmokers before pregnancy was $27.6 \%$ and $57.4 \%$, respectively. $4.1 \%$ of the participants reported to have stopped smoking before pregnancy, whereas about $41 \%$ quitted along pregnancy, resulting in a smoking prevalence at birth of $14.6 \%$. Exposure to ETS also decreased throughout pregnancy to $49.8 \%$ at birth. Lower educational level was significantly associated with both higher smoking prevalence and exposure to ETS and lower smoking cessation. This study demonstrates that smoking and ETS exposure during pregnancy remains high, and that there are still significant socioeconomic inequalities in smoking; thus tobacco-focused preventive interventions need to be reinforced.

Worldwide, smoking is the leading preventable cause of human morbidity and mortality ${ }^{1}$. Consumption of tobacco products account for nearly 8 million deaths per year around the world ${ }^{1}$, and over 10,000 deaths in Portugal $^{2}$.

According to the World Health Organization (WHO) statistics, the prevalence of smoking is still a major public health concern among women ${ }^{3}$. In Portugal, daily smoking remains higher in men than in women, even though prevalence has been decreasing in men (28.8\% in 2005 and $26.7 \%$ in 2014) and increasing in women $(11.3 \% \text { and } 14.6 \% \text { in } 2005 \text { and } 2014 \text {, respectively })^{4}$, these trends are expected to maintain in the next years, in some countries, diminishing the differences observed between sexes ${ }^{1}$.

Soon after the recognition of the health effects related to smoking, it has become evident that exposure to environmental tobacco smoke (ETS) also constitutes a health risk factor ${ }^{5}$ and that there is no secure threshold of exposure $^{6}$. For this reason, ETS was classified as carcinogenic for humans (group 1) in 2012 by the International Agency for Research on Cancer ${ }^{7}$. Available data on ETS exposure shows that, in 2015, 12.8\% of the Portuguese population was exposed to ETS either at home, in transports, public areas or in the workplace more than one hour per day ${ }^{8}$. A substantially higher prevalence (29.0\%) was reported among non-smoking individuals considering only exposure in workplaces and leisure areas in a multi-country European study?.

Portugal was one of the countries that signed the WHO Framework Convention on Tobacco Control, leading to the implementation of the anti-smoking law in all enclosed public spaces, the $37 / 2007 \mathrm{law}^{10}$, in 2008 . This law contained a new framework to protect individuals from ETS, and to encourage cutting down/quitting smoking

${ }^{1}$ Environmental Health Department, National Institute of Health, Rua Alexandre Herculano 321, 4000-055 Porto, Portugal. ${ }^{2} E$ PIUnit - Instituto de Saúde Pública, Universidade do Porto, Rua das Taipas 135, 4050-600 Porto, Portugal. ${ }^{3}$ ICBAS-Institute of Biomedical Sciences Abel Salazar, University of Porto, Rua de Jorge Viterbo Ferreira 228, 4050-313 Porto, Portugal. ${ }^{4}$ These authors contributed equally: Joana Madureira and Alexandra Camelo. ${ }^{凶}$ email:.jpft12@gmail.com 
and establish further regulation for the information provided on tobacco products, packaging, and labeling, as well as further restrictions on the advertising. Despite this and the extensive information on the harmful health effects, some women continue to smoke during pregnancy ${ }^{11-13}$. It has been estimated that $4.2-18.9 \%$ of women in 15 European countries continue smoking during pregnancy ${ }^{13}$. De Wolff et al. ${ }^{11}$ reported a prevalence of maternal smoking in Denmark ranging from 12-22\% before pregnancy to 5-10\% during the pregnancy. These results are in line with prior studies carried out in Finland ${ }^{12}$, Norway ${ }^{14}$, and Iceland ${ }^{15}$. In Portugal, estimates from 2005-2006 indicated that 23\% of Portuguese women smoked early in pregnancy while $12.0 \%$ continued to smoke during pregnancy ${ }^{16}$; more recent data (2017-2018) pointed to a prevalence of $14 \%$ of smoking women during pregnancy ${ }^{17}$. No data is yet available for ETS exposure among pregnant women in Portugal.

There is abundant evidence that patterns of tobacco exposure (use and/or ETS) are related to indicators of socioeconomic position (SEP), not only in the general population ${ }^{18-21}$ but also among pregnant women ${ }^{11,13,22-26}$. These indicators include education ${ }^{11,13,18,21,23,24}$; income ${ }^{19-21}$ and employment ${ }^{11,13,26}$. Moreover, the level of deprivation in the immediate neighborhood of residence seems to play a role in shaping smoking behavior ${ }^{27}$, underlining the existence of residential contextual effects on health related-behavioral practices.

However, given the heterogeneity in smoking prevalence across countries and regions, findings from international studies might not reflect the same patterns found in other countries, particularly in Portugal. In addition, the lack of uniform study design, data collection and study population makes it difficult to assess whether these SEP indicators for smoking and ETS exposure in pregnancy are similar in frequency and effects across countries. To fill this gap and to contribute to monitoring of smoking and ETS exposure trends and other dimensions of smoking, this study assesses smoking prevalence, smoking cessation rate and ETS exposure, as well as the role of the SEP on these behaviors among pregnant women.

\section{Material and methods}

Study design. Data were collected in the framework of the NeoGene project (funded by FCT/FAPESP; reference FAPESP/19914/2014), a cross-sectional birth study that aims to investigate the genetic and epigenetic effects of prenatal tobacco use and ETS exposure, considering simultaneous exposure to numerous and possibly interacting chemicals. In that respect, a wide spectrum of metals, particulate matter, polycyclic aromatic hydrocarbons and other organic compounds assume particular interest.

Participants. The study population included pregnant women receiving prenatal care in Centro Hospitalar de São João (CHSJ). Inclusion criteria comprised women of all ages that spoke Portuguese and singleton pregnancy. Women with infectious diseases, mental and physical disabilities were excluded as well as pregnancies with fetal congenital malformations and genetic disorders. Pregnant women were invited to participate in the project between April 2017 to July 2018 during the last prenatal appointment (after week 36 of pregnancy) at CHSJ. A total of 838 pregnant women were recruited.

Data collection and variable description. Data were collected within $72 \mathrm{~h}$ after delivery, during hospital stay, through individual face-to-face interviews using a paper-based structured questionnaire that was based on the previously used in different Portuguese epidemiological survey ${ }^{16}$. Out of the recruited women, 622 answered the questionnaire; from these, 3 participants were excluded given the missing information in smoking habits and ETS exposure, leaving data from 619 mothers for further analyses.

Demographic variables. Maternal characteristics were collected, including age, parity, area of residence and alcohol drinking habits. Age was categorized as $\leq 25$ years old (young adulthood) and $>25$ years old (mid-age) ${ }^{28}$, in the light of the social age concept; and parity was classified as the number of births for a given woman, counting a multiple birth pregnancy as one; stillbirths were not considered ${ }^{29}$. Participants' home addresses were georeferenced using ArcGIS Online World Geocoding Service and Google Earth, due to their superior positional accuracy $^{30}$. Coordinates were used to classify the area of residence as urban, sub-urban, and rural, using the Portuguese National Institute of Statistics ${ }^{31}$ and to identify the neighborhood deprivation. Sub-urban and rural areas were combined in a single category designated of sub-urban/rural.

Finally, alcohol consumption was classified as "Yes" if women reported alcoholic beverages intake, during pregnancy or in the 3 months preceding pregnancy, even if occasionally.

Socioeconomic variables. Socioeconomic status, defined as maternal educational level, working status and neighborhood deprivation was estimated. Educational level was categorized as 9 years or less, 10-12, and 13 years of education or higher according to the Portuguese education system. In line with an earlier study ${ }^{16}$, maternal working status at the time of the survey was classified as student, housewife, unemployed and employed. For employed women, occupations were then classified as manual or non-manual ${ }^{32}$. Housewives and unemployed were combined in a single category due to the small number of housewife participants' after confirmation that these groups were homogeneous for study variables (smoking, smoking cessation, and ETS exposure). Regarding neighborhood deprivation, herein, an updated version of the European Deprivation Index for Portuguese small-areas (EDI-PT) was used ${ }^{33}$. Briefly, this indicator is based on data from the 2011 European Union-Statistics on Income and Living Conditions Survey (EU-SILC) making use of variables that were common to both the EU-SILC and the 2011 Portuguese Population and Housing census. The EDI-PT was established for the smallest area unit (neighborhood, with an average population of 584 inhabitants) and resulted from the weighted sum of the eight selected variables. Neighborhood deprivation was categorized using tertiles: tertile 1 (least deprived); tertile 2 (medium deprived) and tertile 3 (most deprived). 
Smoking and environmental tobacco smoke exposure. In this study, women were assumed as current smokers if they reported smoking at least one cigarette (or any other smoking products) per day; and considered never smokers if they reported never smoking in their lifetime. Former smokers were defined as those who reported smoking in their lifetime, but who had quit smoking ${ }^{34}$. Further information on the age of smoking initiation and timing of cessation (for former smokers) were also obtained. Smoking status was defined for two different time points: before pregnancy and at birth. At pregnancy, mothers were defined as former smoker if cessation occurred at least 3 months before their pregnancy. Continuation of smoking into pregnancy was analyzed on the basis of cessation in each considered trimester ${ }^{35}$; for this, three additional categories were appraised: (1) quit in the $1^{\text {st }}$ trimester; (2) quit in the $2^{\text {nd }}$ trimester and (3) quit in the $3^{\text {rd }}$ trimester. All those quitting during pregnancy were considered former smokers at birth.

Based on existing knowledge there is no standard procedure for assessment ETS exposure. Thus, in the current study, never-smokers and former smokers were asked to report the duration and frequency of exposure to ETS at home, work and leisure places in the 3 months prior to the pregnancy, and in the $1^{\text {st }}, 2^{\text {nd }}$ and $3^{\text {rd }}$ trimesters. Response options included: "Never"; "Sporadically"; "Daily, less than 1 h"; "Daily, 1-3 h"; "Daily, 3 or more hours". For statistical analyses, responses were dichotomized as "Yes" and "No" and participants were further classified as exposed to ETS and not exposed to ETS, respectively.

Statistical analysis. Results are expressed as means and standard deviations, or as counts and percentages. The distribution of smoking, smoking cessation and ETS exposure according to maternal SEP indicators was examined using the chi-square test or likelihood ratio test, whenever more than $20 \%$ of the frequencies found in the crosstab were below 5. Distribution of age of smoking initiation was analyzed using non-parametric Kruskal-Wallis test since data did not assume a normal distribution. A $p$-value $<0.05$ was considered statistically significant.

Binary logistic regression analysis was used to explore the prediction of smoking at birth, smoking cessation and ETS exposure in the $3^{\text {rd }}$ trimester among pregnant women using both demographic and SEP variables. First, univariate logistic regression models were used for each outcome variable. Variables that proved to be significant at the univariate analysis $(p<0.05)$ were considered for a multivariate logistic regression analysis, in which significant indicators were entered simultaneously. No significant interactions were found between smoking and smoking cessation, and ETS exposure and SEP variables. Associations were presented as crude and adjusted odds ratio (OR) with $95 \%$ confidence intervals $(95 \% \mathrm{CI})$. All analyses were conducted using the Statistical Package for Social Sciences (SPSS, version 26).

Ethical approval and data protection. The project was approved by the Ethical Committee of CHSJ, Porto, Portugal (reference $n^{\circ} .326 / 16$ ) and was conducted in compliance with the Declaration of Helsinki and its amendments. Before enrolment, each participant received detailed information about the project objectives and risks and benefits of their participation. All those that agreed to participate provided written informed consent. All data were handled and stored anonymously.

\section{Results}

Study population characteristics. A total of 619 pregnant women were included in this study. Table 1 summarizes the main characteristics of the studied population. At the time of interview, the majority of pregnant women were older than 25 years old (mean age $=31.4$ years; range: $18-46)$, and $45.2 \%(n=280)$ of them already had children. More participants lived in urban areas than in sub-urban/rural areas (95.0\% vs. 5.0\%). Only 78 women (12.6\%) of the 619 respondents reported drinking at least some alcohol during pregnancy or in the 3 preceding months.

The proportions of mothers with 9 years or less, 10-12 years and at least 13 years of school education were $25.7 \%, 35.4 \%$ and $38.9 \%$, respectively. Among all participants, most of them were employed (77.4\% of the total; of these, $44.1 \%$ in manual and $55.9 \%$ in non-manual jobs). Neighborhood deprivation was assessed according to their level of socioeconomic deprivation (least-medium-most deprived); approximately one-third of the participants resided in the each of these levels of deprivation.

Prevalence of smoking, smoking cessation and ETS exposure. Table 2 indicates that before pregnancy, out of the 619 women who participated in this study, $366(59.1 \%)$ women had never been smokers, $13.3 \%$ $(n=82)$ reported to be former smokers, and $171(27.6 \%)$ women were smokers. At birth, the number of current smokers had decreased to $90(14.6 \%)$ participants. Notably, cigarettes were the only tobacco product consumed by smoking women.

Data also shows that cessation occurred before pregnancy for $4.1 \%$ of smokers $(n=7)$, and during pregnancy for $40.6 \%$ of them $(n=70)$. To note that, $90(52.3 \%)$ women have never stopped tobacco use.

Concurrently, the prevalence of ETS exposure among non-smoking pregnant women (including former smokers) was found to decrease from $57.4 \%$ before pregnancy to $51.2 \%$ in the $1^{\text {st }}$ trimester, $51.4 \%$ in the $2^{\text {nd }}$ trimester, and $49.8 \%$ in the $3^{\text {rd }}$ trimester; this represents an increment in the number of women who reported no ETS exposure at home, leisure places or at work, along the three trimesters of pregnancy.

Self-reported smoking, smoking cessation and ETS exposure by educational level, working status and neighborhood deprivation is presented in Table 2. The average age of smoking initiation was found to be higher as maternal educational level increased $(p<0.001)$. Likewise, early smoking initiation varied significantly according to working status $(p<0.001)$; the students and housewives/unemployed being the earlier initiators. No differences were observed for neighborhood deprivation $(p=0.768)$. 


\begin{tabular}{|c|c|c|}
\hline & $\mathbf{N}$ & $\%$ \\
\hline Age [years $($ mean \pm SD, min.-max.)] & 619 & $31.4 \pm 5.6(18-46)$ \\
\hline$\leq 25$ & 106 & 17.1 \\
\hline$>25$ & 513 & 82.9 \\
\hline \multicolumn{3}{|l|}{ Parity* } \\
\hline 0 & 338 & 54.6 \\
\hline$\geq 1$ & 280 & 45.2 \\
\hline \multicolumn{3}{|l|}{ Area of residence ${ }^{* *}$} \\
\hline Sub-urban/rural area & 31 & 5.0 \\
\hline Urban area & 586 & 95.0 \\
\hline \multicolumn{3}{|l|}{ Alcohol consumption } \\
\hline No & 541 & 87.4 \\
\hline Yes & 78 & 12.6 \\
\hline \multicolumn{3}{|l|}{ Educational level } \\
\hline $0-9$ years & 159 & 25.7 \\
\hline $10-12$ years & 219 & 35.4 \\
\hline$\geq 13$ years & 241 & 38.9 \\
\hline \multicolumn{3}{|l|}{ Working status } \\
\hline Unemployed/housewives & 128 & 20.7 \\
\hline Students & 12 & 1.9 \\
\hline Employed & 479 & 77.4 \\
\hline Manual & 211 & 44.1 \\
\hline Non-manual & 268 & 55.9 \\
\hline \multicolumn{3}{|l|}{ Neighborhood deprivation ${ }^{* * *}$} \\
\hline Least deprived & 198 & 32.0 \\
\hline Medium deprived & 199 & 32.1 \\
\hline Most deprived & 198 & 32.0 \\
\hline
\end{tabular}

Table 1. Characteristics of pregnant women included in the study $(n=619) .{ }^{\star} 1$ missing value; ${ }^{\star *} 2$ missing values; ${ }^{* *} 24$ missing values.

The distribution of never smokers, former smokers and current smokers, both before pregnancy and at birth, was also found to be significantly different depending on the educational level $(p<0.001)$ and working status $(p<0.001)$; the majority of smoking pregnant women were found to have lower educational levels $(<13$ years $)$ and were either working on a manual job or were unemployed/housewives. No differences were observed regarding neighborhood deprivation before pregnancy $(p=0.550)$. As expected, since women did not move to a new home, similar results were obtained at birth $(p=0.638)$. Concerning smoking cessation, continuers and quitters significantly differed in educational level $(p<0.012)$, but not in working status $(p=0.257)$ or neighborhood deprivation ( $p=0.144$ ), being those who had more than 13 years of education more likely to quit smoking compared with mothers who had been educated for less than 12 years.

Self-reported ETS exposure among never and former smokers differed significantly according to educational level at all-time points considered ( $p=0.004$ before pregnancy and $p<0.001$ throughout pregnancy) and working status during pregnancy ( $p=0.049$ in the $1^{\text {st }}, p=0.042$ in the $2^{\text {nd }}$ and $p=0.022$ in the $3^{\text {rd }}$ trimester); women with higher educational level and working in non-manual jobs were found to be less exposed. Detailed information on the duration and frequency of ETS exposure throughout pregnancy is presented in Table 1S.

Associations between demographic and SEP variables and smoking. Table 3 presents the results of unadjusted and adjusted models predicting maternal smoking from demographic and SEP variables. The results obtained with the model adjusted for variables found to be significant at the univariate model show a significant association between age and smoking (OR 0.554; 95\%CI 0.314-0.978) as well as between educational level and smoking (OR 0.488; 95\%CI 0.286-0.831 for 10-12 years, and OR 0.156; 95\%CI 0.066-0.371 for more than 13 years).

Associations between demographic and SEP variables and ETS exposure. According to findings presented in Table 3, in the adjusted model, those with less than 13 years of education were more likely to be exposed to ETS. Thus, university or equivalent level as the highest educational level (OR 0.397; 95\%CI $0.218-0.729$ ) was associated with decreased odds of exposure to ETS.

Associations between demographic and SEP variables and smoking cessation. Table 4 summarizes the results of unadjusted and adjusted analyses to characterize smoking cessation by demographic and SEP variables. In line with the observed for smoking and ETS exposure, adjusted logistic regression model showed 


\begin{tabular}{|c|c|c|c|c|c|c|c|c|c|c|c|c|c|c|}
\hline & \multirow[b]{3}{*}{ Total } & \multicolumn{3}{|c|}{ Educational level } & \multirow[b]{3}{*}{$p$ value } & \multicolumn{4}{|c|}{ Working status } & \multirow[b]{3}{*}{$p$ value } & \multicolumn{3}{|c|}{ Neighborhood deprivation } & \multirow[b]{3}{*}{ p value } \\
\hline & & & & & & \multirow[b]{2}{*}{$\begin{array}{l}\text { Housewives/ } \\
\text { unemployed }\end{array}$} & \multirow[b]{2}{*}{ Students } & \multicolumn{2}{|l|}{ Employed } & & \multirow[b]{2}{*}{$\begin{array}{l}\text { Least } \\
\text { deprived }\end{array}$} & \multirow[b]{2}{*}{$\begin{array}{l}\text { Medium } \\
\text { deprived }\end{array}$} & \multirow[b]{2}{*}{$\begin{array}{l}\text { Most } \\
\text { deprived }\end{array}$} & \\
\hline & & $0-9$ years & $\begin{array}{l}10- \\
12 \text { years }\end{array}$ & $\geq 13$ years & & & & Manual & \begin{tabular}{|l|}
$\begin{array}{l}\text { Non- } \\
\text { manual }\end{array}$ \\
\end{tabular} & & & & & \\
\hline \multicolumn{15}{|l|}{ Smoking } \\
\hline \multicolumn{15}{|c|}{ Smoking initiation* } \\
\hline $\begin{array}{l}\text { Age } \\
\text { (years) }\end{array}$ & $16.5 \pm 3.5$ & $15.8 \pm 3.4$ & $16.3 \pm 3.3$ & $17.8 \pm 3.7$ & $<0.001$ & $15.5 \pm 3.6$ & $15.0 \pm 1.8$ & $16.6 \pm 3.3$ & $17.3 \pm 3.6$ & $<0.001$ & $16.8 \pm 3.7$ & $16.4 \pm 3.5$ & $16.4 \pm 3.5$ & 0.768 \\
\hline \multicolumn{15}{|c|}{ Smoking status before pregnancy } \\
\hline $\begin{array}{l}\text { Never } \\
\text { smokers }\end{array}$ & \begin{tabular}{|l|}
366 \\
$(59.1 \%)$
\end{tabular} & \begin{tabular}{|l|}
72 \\
$(19.7 \%)$
\end{tabular} & $\begin{array}{l}121 \\
(33.0 \%)\end{array}$ & $\begin{array}{l}173 \\
(47.3 \%)\end{array}$ & \multirow{3}{*}{$<0.001$} & $60(16.4 \%)$ & $8(2.2 \%)$ & $\begin{array}{l}119 \\
(32.5 \%)\end{array}$ & \begin{tabular}{|l}
179 \\
$(48.9 \%)$
\end{tabular} & \multirow{3}{*}{$<0.001$} & $\begin{array}{l}122 \\
(35.2 \%)\end{array}$ & $\begin{array}{l}118 \\
(34.0 \%)\end{array}$ & $\begin{array}{l}107 \\
(30.8 \%)\end{array}$ & \multirow{3}{*}{0.550} \\
\hline $\begin{array}{l}\text { Former } \\
\text { smokers }\end{array}$ & $\begin{array}{l}82 \\
(13.3 \%) \\
\end{array}$ & $\begin{array}{l}19 \\
(23.2 \%) \\
\end{array}$ & $\begin{array}{l}28 \\
(34.1 \%) \\
\end{array}$ & $35(42.7 \%)$ & & $14(17.1 \%)$ & $1(1.2 \%)$ & $\begin{array}{l}26 \\
(31.7 \%) \\
\end{array}$ & $\begin{array}{l}41 \\
(50.0 \%) \\
\end{array}$ & & \begin{tabular}{|l|}
21 \\
$(27.2 \%)$ \\
\end{tabular} & $\begin{array}{l}26 \\
(33.8 \%)\end{array}$ & $\begin{array}{l}30 \\
(39.0 \%)\end{array}$ & \\
\hline $\begin{array}{l}\text { Current } \\
\text { smokers }\end{array}$ & \begin{tabular}{|l|}
171 \\
$(27.6 \%)$
\end{tabular} & \begin{tabular}{|l|}
68 \\
$(39.8 \%)$ \\
\end{tabular} & \begin{tabular}{|l|}
70 \\
$(40.9 \%)$ \\
\end{tabular} & $33(19.3 \%)$ & & $54(31.6 \%)$ & $3(1.7 \%)$ & \begin{tabular}{|l|}
66 \\
$(38.6 \%)$ \\
\end{tabular} & $\begin{array}{l}48 \\
(28.1 \%)\end{array}$ & & $\begin{array}{l}55 \\
(32.2 \%)\end{array}$ & $\begin{array}{l}55 \\
(32.2 \%)\end{array}$ & $\begin{array}{l}61 \\
(35.6 \%)\end{array}$ & \\
\hline \multicolumn{15}{|c|}{ Smoking status at birth } \\
\hline $\begin{array}{l}\begin{array}{l}\text { Never } \\
\text { smokers }\end{array} \\
\end{array}$ & \begin{tabular}{|l|}
366 \\
$(59.1 \%)$
\end{tabular} & \begin{tabular}{|l|}
72 \\
$(19.7 \%)$ \\
\end{tabular} & $\begin{array}{l}121 \\
(33.1 \%)\end{array}$ & $\begin{array}{l}173 \\
(47.2 \%)\end{array}$ & \multirow{3}{*}{$<0.001$} & $60(16.4 \%)$ & $8(2.2 \%)$ & $\begin{array}{l}119 \\
(32.5 \%)\end{array}$ & \begin{tabular}{|l|}
179 \\
$(48.9 \%)$ \\
\end{tabular} & \multirow{3}{*}{$<0.001$} & $\begin{array}{l}122 \\
(35.2 \%)\end{array}$ & $\begin{array}{l}118 \\
(34.0 \%)\end{array}$ & $\begin{array}{l}107 \\
(30.8 \%)\end{array}$ & \multirow{3}{*}{0.638} \\
\hline $\begin{array}{l}\text { Former } \\
\text { smokers }\end{array}$ & $\begin{array}{l}163 \\
(26.3 \%)\end{array}$ & $\begin{array}{l}42 \\
(25.8 \%)\end{array}$ & $\begin{array}{l}64 \\
(39.3 \%)\end{array}$ & $57(34.9 \%)$ & & $34(20.9 \%)$ & $2(1.2 \%)$ & $\begin{array}{l}56 \\
(34.4 \%)\end{array}$ & $\begin{array}{l}71 \\
(43.5 \%)\end{array}$ & & $\begin{array}{l}48 \\
(30.4 \%)\end{array}$ & $\begin{array}{l}51 \\
(32.3 \%)\end{array}$ & $\begin{array}{l}59 \\
(37.3 \%)\end{array}$ & \\
\hline $\begin{array}{l}\text { Current } \\
\text { smokers }\end{array}$ & $\begin{array}{l}90 \\
(14.6 \%)\end{array}$ & \begin{tabular}{|l}
45 \\
$(50.0 \%)$
\end{tabular} & $\begin{array}{l}34 \\
(37.8 \%)\end{array}$ & $11(12.2 \%)$ & & $34(37.8 \%)$ & $2(2.2 \%)$ & $\begin{array}{l}36 \\
(40.0 \%)\end{array}$ & $\begin{array}{l}18 \\
(20.0 \%)\end{array}$ & & $\begin{array}{l}28 \\
(31.1 \%)\end{array}$ & $\begin{array}{l}30 \\
(33.3 \%)\end{array}$ & $\begin{array}{l}32 \\
(35.6 \%)\end{array}$ & \\
\hline \multicolumn{15}{|c|}{ Cessation rate ${ }^{* *}$} \\
\hline $\begin{array}{l}\text { No cessa- } \\
\text { tion }\end{array}$ & \begin{tabular}{|l|}
90 \\
$(52.3 \%)$ \\
\end{tabular} & \begin{tabular}{|l|}
45 \\
$(50.0 \%)$ \\
\end{tabular} & \begin{tabular}{|l|}
34 \\
$(37.8 \%)$ \\
\end{tabular} & $11(12.2 \%)$ & \multirow{5}{*}{$0.012^{*}$} & $34(37.8 \%)$ & $2(2.2 \%)$ & \begin{tabular}{|l|}
36 \\
$(40.0 \%)$ \\
\end{tabular} & \begin{tabular}{|l|l|}
18 \\
$(20.0 \%)$ \\
\end{tabular} & & \begin{tabular}{|l|}
28 \\
$(31.1 \%)$ \\
\end{tabular} & $\begin{array}{l}30 \\
(33.3 \%) \\
\end{array}$ & $\begin{array}{l}32 \\
(35.6 \%) \\
\end{array}$ & \\
\hline $\begin{array}{l}\text { Before } \\
\text { pregnancy }\end{array}$ & $7(4.1 \%)$ & $2(28.6 \%)$ & $2(28.6 \%)$ & $3(42.8 \%)$ & & $1(14.3 \%)$ & 0 & $2(28.6 \%)$ & $4(57.1 \%)$ & & $1(14.2 \%)$ & $3(42.9 \%)$ & $3(42.9 \%)$ & \\
\hline $\begin{array}{l}1^{\text {st }} \text { trimes- } \\
\text { ter }\end{array}$ & $\begin{array}{l}59 \\
(34.3 \%) \\
\end{array}$ & \begin{tabular}{|l|l|}
15 \\
$(25.4 \%)$ \\
\end{tabular} & $\begin{array}{l}26 \\
(44.1 \%)\end{array}$ & $18(30.5 \%)$ & & $13(22.0 \%)$ & $1(1.7 \%)$ & $\begin{array}{l}22 \\
(37.3 \%)\end{array}$ & $\begin{array}{l}23 \\
(39.0 \%)\end{array}$ & $0.257^{\#}$ & \begin{tabular}{|l|}
23 \\
$(39.0 \%)$ \\
\end{tabular} & $\begin{array}{l}15 \\
(25.4 \%)\end{array}$ & $\begin{array}{l}21 \\
(35.6 \%)\end{array}$ & $0.144^{*}$ \\
\hline $\begin{array}{l}2^{\text {nd }} \\
\text { trimester }\end{array}$ & $8(4.6 \%)$ & $5(62.5 \%)$ & $3(37.5 \%)$ & 0 & & $4(50.0 \%)$ & 0 & $3(37.5 \%)$ & $1(12.5 \%)$ & & 0 & $4(50.0 \%)$ & $4(50.0 \%)$ & \\
\hline $\begin{array}{l}3^{\text {rd }} \text { trimes- } \\
\text { ter }\end{array}$ & $3(1.7 \%)$ & $1(33.3 \%)$ & $2(66.7 \%)$ & 0 & & $1(33.3 \%)$ & 0 & $2(66.7 \%)$ & 0 & & $1(33.3 \%)$ & $2(66.7 \%)$ & 0 & \\
\hline Exposure to & o ETS ${ }^{* * *}$ & & & & & & & & & & & & & \\
\hline Before pregn & nancy & & & & & & & & & & & & & \\
\hline No & \begin{tabular}{|l|}
191 \\
$(42.6 \%)$
\end{tabular} & $\begin{array}{l}32 \\
(16.8 \%)\end{array}$ & \begin{tabular}{|l|}
53 \\
$(27.7 \%)$
\end{tabular} & $\begin{array}{l}106 \\
(55.5 \%)\end{array}$ & O & $27(14.1 \%)$ & $4(2.1 \%)$ & \begin{tabular}{|l|}
54 \\
$(28.3 \%)$
\end{tabular} & $\begin{array}{l}106 \\
(55.5 \%)\end{array}$ & 120 & \begin{tabular}{|l|}
62 \\
$(34.1 \%)$
\end{tabular} & $\begin{array}{l}66 \\
(36.2 \%)\end{array}$ & $\begin{array}{l}54 \\
(29.7 \%)\end{array}$ & 0550 \\
\hline Yes & $\begin{array}{l}257 \\
(57.4 \%)\end{array}$ & $\begin{array}{l}59 \\
(23.0 \%)\end{array}$ & \begin{tabular}{|l|}
96 \\
$(37.3 \%)$ \\
\end{tabular} & $\begin{array}{l}102 \\
(39.7 \%)\end{array}$ & 0.004 & $47(18.3 \%)$ & $5(1.9 \%)$ & $\begin{array}{l}91 \\
(35.4 \%)\end{array}$ & $\begin{array}{l}114 \\
(44.4 \%)\end{array}$ & 0.100 & \begin{tabular}{|l|}
81 \\
$(33.5 \%)$ \\
\end{tabular} & $\begin{array}{l}78 \\
(32.2 \%)\end{array}$ & $\begin{array}{l}83 \\
(34.3 \%)\end{array}$ & 0.500 \\
\hline 1st trimester & & & & & & & & & & & & & & \\
\hline No & \begin{tabular}{|l|}
222 \\
$(48.8 \%)$
\end{tabular} & $\begin{array}{l}37 \\
(16.7 \%)\end{array}$ & \begin{tabular}{|l|}
60 \\
$(27.0 \%)$
\end{tabular} & $\begin{array}{l}125 \\
(56.3 \%)\end{array}$ & 0001 & $30(13.5 \%)$ & $4(1.8 \%)$ & $\begin{array}{l}64 \\
(28.8 \%)\end{array}$ & $\begin{array}{l}124 \\
(55.9 \%)\end{array}$ & 0049 & $\begin{array}{l}68 \\
(32.4 \%)\end{array}$ & $\begin{array}{l}80 \\
(38.1 \%)\end{array}$ & $\begin{array}{l}62 \\
(29.5 \%)\end{array}$ & م 208 \\
\hline Yes & $\begin{array}{l}233 \\
(51.2 \%)\end{array}$ & $\begin{array}{l}56 \\
(24.0 \%)\end{array}$ & $\begin{array}{l}91 \\
(39.1 \%)\end{array}$ & $86(36.9 \%)$ & $<0.001$ & $45(19.3 \%)$ & $5(2.2 \%)$ & $\begin{array}{l}83 \\
(35.6 \%)\end{array}$ & $\begin{array}{l}100 \\
(42.9 \%)\end{array}$ & 0.049 & $\begin{array}{l}76 \\
(34.4 \%)\end{array}$ & $\begin{array}{l}67 \\
(30.3 \%)\end{array}$ & $\begin{array}{l}78 \\
(35.3 \%)\end{array}$ & 0.208 \\
\hline 2nd trimeste & & & & & & & & & & & & & & \\
\hline No & \begin{tabular}{|l|}
250 \\
$(48.6 \%)$ \\
\end{tabular} & \begin{tabular}{|l|}
41 \\
$(16.4 \%)$
\end{tabular} & \begin{tabular}{|l|}
74 \\
$(29.6 \%)$ \\
\end{tabular} & $\begin{array}{ll}135 \\
(54.0 \%)\end{array}$ & $<0001$ & $36(14.4 \%)$ & $5(2.0 \%)$ & $\begin{array}{l}73 \\
(29.2 \%)\end{array}$ & \begin{tabular}{|l|}
136 \\
$(54.4 \%)$
\end{tabular} & 0042 & $\begin{array}{l}81 \\
(34.2 \%)\end{array}$ & $\begin{array}{l}87 \\
(36.7 \%)\end{array}$ & $\begin{array}{l}69 \\
(29.1 \%)\end{array}$ & 0149 \\
\hline Yes & $\begin{array}{l}264 \\
(51.4 \%)\end{array}$ & \begin{tabular}{|l|}
67 \\
$(25.4 \%)$
\end{tabular} & $\begin{array}{l}103 \\
(39.0 \%)\end{array}$ & $95(35.6 \%)$ & $<000$ & $52(19.7 \%)$ & $5(1.9 \%)$ & $\begin{array}{l}96 \\
(36.4 \%)\end{array}$ & $\begin{array}{l}111 \\
(52.0 \%)\end{array}$ & 0.042 & \begin{tabular}{|l|}
86 \\
$(34.0 \%)$
\end{tabular} & $\begin{array}{l}75 \\
(29.6 \%)\end{array}$ & $\begin{array}{l}92 \\
(36.4 \%)\end{array}$ & 0.149 \\
\hline $3 r d$ trimester & & & & & & & & & & & & & & \\
\hline No & \begin{tabular}{|l}
262 \\
$(50.2 \%)$
\end{tabular} & $\begin{array}{l}44 \\
(16.8 \%)\end{array}$ & \begin{tabular}{|l|}
77 \\
$(29.4 \%)$
\end{tabular} & $\begin{array}{l}141 \\
(53.8 \%)\end{array}$ & & $41(15.7 \%)$ & $5(1,9 \%)$ & $\begin{array}{l}74 \\
(28.2 \%)\end{array}$ & $\begin{array}{l}142 \\
(54.2 \%)\end{array}$ & & $\begin{array}{l}86 \\
(34.7 \%)\end{array}$ & $\begin{array}{l}92 \\
(37.1 \%)\end{array}$ & $\begin{array}{l}70 \\
(28.2 \%)\end{array}$ & \\
\hline Yes & $\begin{array}{l}260 \\
(49.8 \%)\end{array}$ & $\begin{array}{l}69 \\
(26.5 \%)\end{array}$ & $\begin{array}{l}103 \\
(39.6 \%)\end{array}$ & $88(33.9 \%)$ & $<0.001$ & $51(19.6 \%)$ & $5(1.9 \%)$ & $\begin{array}{l}98 \\
(37.7 \%)\end{array}$ & $\begin{array}{l}106 \\
(40.8 \%)\end{array}$ & 0.022 & $\begin{array}{l}81 \\
(32.4 \%)\end{array}$ & $\begin{array}{l}74 \\
(29.6 \%)\end{array}$ & $\begin{array}{l}95 \\
(38.0 \%)\end{array}$ & 0.053 \\
\hline
\end{tabular}

Table 2. Self-reported maternal smoking, smoking cessation and exposure to environmental tobacco smoke (ETS) according to educational level, working status and neighborhood deprivation. Row percentages are presented in all cases, except for total, that indicates column percentages. ${ }^{*}$ Mean $\pm \mathrm{SD} ;{ }^{* *} 4$ missing values; ${ }_{\star * \star}$ ETS exposure was calculated considering non-smokers and former smokers at each time point. "Likelihood ratio test instead of chi-square test. 


\begin{tabular}{|c|c|c|c|c|}
\hline & \multicolumn{2}{|l|}{ Smoking } & \multicolumn{2}{|l|}{ ETS exposure } \\
\hline & OR $(95 \% \mathrm{CI})$ & aOR $(95 \% \mathrm{CI})^{\varsigma}$ & OR $(95 \% \mathrm{CI})$ & aOR $(95 \% \mathrm{CI})^{\mathrm{Y}}$ \\
\hline \multicolumn{5}{|l|}{ Maternal age } \\
\hline$\leq 25$ & Reference & Reference & Reference & - \\
\hline$>25$ & $0.336(0.203-0.554)^{\star *}$ & $0.554(0.314-0.978)^{*}$ & $0.621(0.379-1.020)$ & - \\
\hline \multicolumn{5}{|l|}{ Parity } \\
\hline 0 & Reference & - & Reference & - \\
\hline$\geq 1$ & $1.123(0.718-1.758)$ & - & $0.811(0.575-1.143)$ & - \\
\hline \multicolumn{5}{|l|}{ Area of residence } \\
\hline Sub-urban/rural area & Reference & - & Reference & - \\
\hline Urban area & $1.627(0.484-5.469)$ & - & $1.382(0.641-2.982)$ & - \\
\hline \multicolumn{5}{|l|}{ Alcohol consumption } \\
\hline No & Reference & - & Reference & - \\
\hline Yes & $1.079(0.558-2.089)$ & - & $1.489(0.882-2.513)$ & - \\
\hline \multicolumn{5}{|l|}{ Educational level } \\
\hline $0-9$ years & Reference & Reference & Reference & Reference \\
\hline $10-12$ years & $0.466(0.282-0.770)^{*}$ & $0.488(0.286-0.831)^{*}$ & $0.882(0.547-1.424)$ & $0.887(0.542-1.450)$ \\
\hline$\geq 13$ years & $0.121(0.060-0.243)^{* *}$ & $0.156(0.066-0.371)^{* *}$ & $0.397(0.250-0.629)^{* *}$ & $0.397(0.218-0.729)^{*}$ \\
\hline \multicolumn{5}{|l|}{ Working status } \\
\hline Unemployed/housewives & Reference & Reference & Reference & Reference \\
\hline Students & $0.553(0.115-2.653)$ & $0.413(0.080-2.133)$ & $0.774(0.210-2.852)$ & $0.802(0.212-3.032)$ \\
\hline Manual & $0.569(0.334-0.968)^{*}$ & $0.576(0.327-1.017)$ & $1.056(0.637-1.751)$ & $0.897(0.533-1.511)$ \\
\hline Non-manual & $0.199(0.107-0.370)^{\star *}$ & $0.591(0.276-1.264)$ & $0.588(0.365-0.949)^{*}$ & $0.920(0.530-1.597)$ \\
\hline \multicolumn{5}{|c|}{ Neighborhood deprivation } \\
\hline Least deprived & Reference & - & Reference & - \\
\hline Medium deprived & $1.078(0.617-1.882)$ & - & $0.857(0.559-1.313)$ & - \\
\hline Most deprived & $1.170(0.675-2.029)$ & - & $1.404(0.913-2.159)$ & - \\
\hline
\end{tabular}

Table 3. Crude and adjusted logistic regression analysis of demographic and socioeconomic status variables on smoking and environmental tobacco smoke (ETS) exposure. OR, odds ratio; aOR, adjusted odds ratio; CI, confidence interval. ${ }^{\star}: p<0.05 ;{ }^{* *}: p<0.001$. ${ }^{\S}$ Adjusted for age, educational level and working status. ${ }^{¥}$ Adjusted for educational level and working status.

that pregnant women with higher educational level were more likely to quit consumption (OR 2.969; $95 \%$ CI 1.037-8.506).

\section{Discussion}

Smoking and ETS exposure are associated with several immediate and long-term health effects on both pregnant women and their newborns ${ }^{5,36}$. Understanding the trends and determinants of these behaviors is crucial for the development of effective preventive interventions that aim to reduce the associated burden of disease. In this context, the role of health professionals assumes particular importance and can constitute an efficacious preventive health action as they can offer the best behavioral counseling and/or support for smoking cessation during, and preferably also after, pregnancy; as well as recommend the creation of a smoke free home, including post-discharge from delivery.

In Portugal, there are no previous studies on ETS exposure during pregnancy, and information available on smoking and smoking cessation among pregnant women has not been reported in detail since 2013, in a study that examines data from 2005 to $2006^{16}$. Meanwhile, Portugal has surpassed different societal and economical challenges, and new legislation to promote tobacco cessation and smoke-free environments has been implemented (namely, Lei $37 / 2007^{10}$ and tax and prices measures, as defined in Decreto-Lei $73 / 2010^{37}$ ). The latest population estimates from 2014 shows that Portuguese women were at that time in stage 2 of the tobacco epidemic model, and, therefore, its peak had not yet been reached ${ }^{4}$.

This study found that the prevalence of smoking before pregnancy $(27.7 \%)$ was higher than in the previous Portuguese study from 2013, where the prevalence was $22.9 \%{ }^{16}$. Even though the most recent estimate on smoking prevalence among Portuguese women has been set in $14.6 \%$, one must keep in mind that our study includes only women of fertile age, and that these are the groups presenting higher prevalence of smokers ${ }^{4}$. Furthermore, women included in this study were mainly from urban areas, a factor that may be associated with increased smoking frequency ${ }^{4}$; some authors have highlighted that social and cultural dimensions of lifestyle could account for rural-urban differences ${ }^{38}$.

At birth, results showed that the prevalence of smoking women was $14.6 \%$, which is very close to what has been recently reported in Portugal $(14 \%)^{17}$, and slightly higher than the described by Alves et al ${ }^{16}(12 \%)$. The prevalence at delivery found in this study is relatively high when compared to other countries, such as Denmark 


\begin{tabular}{|c|c|c|}
\hline & OR $(95 \% \mathrm{CI})$ & aOR $(95 \% \mathrm{CI})^{5}$ \\
\hline \multicolumn{3}{|l|}{ Maternal age } \\
\hline$\leq 25$ & Reference & - \\
\hline$>25$ & $1.795(0.896-3.596)$ & - \\
\hline \multicolumn{3}{|l|}{ Parity } \\
\hline 0 & Reference & - \\
\hline$\geq 1$ & $0.639(0.343-1.193)$ & - \\
\hline \multicolumn{3}{|l|}{ Area of residence } \\
\hline Sub-urban/rural area & Reference & - \\
\hline Urban area & $2.651(0.270-26.026)$ & - \\
\hline \multicolumn{3}{|l|}{ Alcohol consumption } \\
\hline No & Reference & - \\
\hline Yes & $1.818(0.807-4.097)$ & - \\
\hline \multicolumn{3}{|l|}{ Educational level } \\
\hline $0-9$ years & Reference & Reference \\
\hline $10-12$ years & $1.857(0.926-3.722)$ & $1.678(0.816-3.451)$ \\
\hline$\geq 13$ years & $3.652(1.505-8.865)^{* *}$ & $2.969(1.037-8.506)^{*}$ \\
\hline \multicolumn{3}{|l|}{ Working status } \\
\hline Unemployed/housewives & Reference & Reference \\
\hline Students & $0.895(0.076-10.528)$ & $1.022(0.085-12.294)$ \\
\hline Manual & $1.483(0.703-3.128)$ & $1.536(0.716-3.295)$ \\
\hline Non-manual & $2.784(1.231-6.295)^{*}$ & $1.786(0.712-4.485)$ \\
\hline \multicolumn{3}{|l|}{ Neighborhood deprivation } \\
\hline Least deprived & Reference & - \\
\hline Medium deprived & $0.864(0.402-1.855)$ & - \\
\hline Most deprived & $0.945(0.449-1.988)$ & - \\
\hline
\end{tabular}

Table 4. Crude and adjusted logistic regression analysis of demographic and socioeconomic status variables on smoking cessation. OR, odds ratio; aOR, adjusted odds ratio; CI, confidence interval. ${ }^{\star} p<0.05 ;{ }^{\star *} p<0.001$. ${ }^{\S}$ Adjusted for educational level.

$(6 \%)^{11}$, USA $(7.1 \%)^{22}$, and China $(3.8 \%)^{25}$. This discrepancy could be attributed to differences in study design, sample selection, variable definition, but also to tobacco control policies of each country, and population awareness for tobacco effects ${ }^{39}$. Herein, more than half of the smoking women (52.3\%) did not stop smoking neither in the 3 months before pregnancy, nor during gestation. In accordance with our findings, Alves et al. ${ }^{16}$, in Portugal, reported a similar cessation rate $(52.6 \%)$, showing that there was no significant progression on this matter in the last 15 years.

In line with prior research on the role of demographic characteristics on maternal consumption in pregnancy ${ }^{11,23,25,40}$, the current study suggests that mid-age women (older than 25 years-old), probably due to individual life circumstances and higher understanding of harms related to smoking, are less likely to smoke. Other relevant predictors of smoking among pregnant women identified in previous studies include parity ${ }^{11,24}$, and alcohol consumption ${ }^{13,23}$. Nevertheless, in our study, after adjustment for significant variables, there was no significant association between parity or alcohol use and smoking status.

As reported by other authors ${ }^{11,13,22}$, in our study, educational disadvantage was found to be a predictor of smoking status during pregnancy; participants who belong to the highest educational levels had a lower probability of smoking. In Portugal, these inequalities in pregnant smoking women have also been previously described $^{16}$. Educational level may not only increase the likelihood of awareness of harmful effects of smoking during pregnancy ${ }^{41}$, but also of access to cessation programs ${ }^{42}$. In fact, several studies have noted that more highly educated women were more likely to quit smoking during pregnancy ${ }^{11,22,43}$. In our findings, a significant association was also found between educational levels (an indicator of SEP) and smoking cessation, indicating that continuers were more often those with lower education and with unskilled work.

In the current study, findings concerning working status, even though not significant, align with those in the literature, that report occupation as a predictor for smoking before and during pregnancy ${ }^{11,13,26}$; occupation is also commonly related to an individual education and income ${ }^{44}$. Research also supports that smoking is a habituated response to the circumstance of housewife, often associated with economic vulnerability ${ }^{45}$.

Altogether, these findings evidence that women maintain their smoking habits even after learning that they are pregnant; however, during pregnancy, women are particularly responsive to accessible tobacco cessation interventions ${ }^{46,47}$, and therefore it is crucial to devote increased efforts on effective targeted smoking prevention and cessation strategies in this period of life, in the frame of the already existing national tobacco control plan and smoking cessation guidelines especially in Portugal.

Another important finding of the current study relates to the characterization of ETS exposure during pregnancy, carried out in Portugal for the first time. Results obtained showed that more than half of non-smokers 
participants reported to be exposed to ETS before pregnancy (57.4\%) and that this number decreases with pregnancy progression. This prevalence estimate is lower than that described by other authors ${ }^{48}$, but higher when compared to a recent study carried out by Alghamdi et al. ${ }^{49}$. In fact, the prevalence here obtained seems high if one considers that in 2015, it was estimated that only $12.8 \%$ of the total Portuguese population reported to be exposed to ETS ${ }^{8}$. In Portugal, a smoke-free law was implemented in 2008 (Lei 37/2007 ${ }^{10}$ ) in order to decrease exposure to ETS and its effects among non-smokers. Nevertheless, residential homes and private vehicles are not covered in the mentioned law, what may justify the high value here observed; these results suggest that ETS exposure may constitute a serious negative health outcome for pregnant women and developing fetus and, thus, it is essential to strengthen people's awareness of active and passive tobacco hazards.

Our results also indicate that exposure to ETS tended to be associated with participants who were less educated, as has been demonstrated by other authors ${ }^{8,49}$; participants with high levels of literacy are more likely to engage in health-promoting behaviors ${ }^{42}$. These data suggest that it is essential to strength people's awareness regarding tobacco hazards and to implement more comprehensive smoke-free laws and legislations to protect particularly the less educated individuals from ETS.

Possible limitations in this study include selection and recall bias, lack of data on income and smoking status of the partner prior and during pregnancy. Another inherent limitation of this study comes from the representativeness of the study population that was not nationally representative. The sample consisted of pregnant women from the Porto Metropolitan Area, in the north of Portugal, which could differ from the general birthing Portuguese population or even from those pregnant women living in other Portuguese geographic regions (urban or non-urban areas). Therefore, the generalizability of the obtained findings should be considered when interpreting the results. By recruiting volunteers to a study on smoking and its health effects, it is more likely to have smokers aware of smoking hazards as participants. In addition, subject categorization as smoker or non-smoker was based on self-reported information; ideally, this should have been confirmed with biomarkers, such as urinary cotinine levels. Nonetheless, qualitative measures are routinely used in many national and international health behavioral surveillance studies, providing accurate results as previously described by e.g. Bernstein et al. ${ }^{50}$ and Mattsson et al. ${ }^{51}$. Recall bias, on the other hand, was kept minimal due to the short-time relapse from pregnancy to data collection. Information on income has not been collected but many studies have shown that this indicator is closely related to education and occupation ${ }^{52}$. In opposition, we have no proxy variable for smoking status of partners, which is known to have a significant influence on continuation ${ }^{23,24}$ and smoking cessation behaviors ${ }^{23}$. In addition, information whether the participants were asked by their health care provider, about advice and/or support on non-pharmacological strategies or pharmacological interventions, or both, to quit smoking should be collected in future epidemiological studies. On the other hand, this is a comprehensive study that not only includes a large number of participants but also contains variables that have not yet been examined before in Portugal, such as data for each trimester of pregnancy, alcohol use and neighborhood deprivation. In addition, by collecting information on ETS exposure in different indoor settings prior or during pregnancy made it possible to explore, for the first time in Portugal, the exposure to ETS among pregnant women.

In conclusion, our findings show that the smoking prevalence and ETS exposure remain a current challenge in Portugal, with $27.6 \%$ of smoking women before pregnancy, $14.6 \%$ of smoking women at delivery and $49.8 \%$ of non-smoking pregnant women exposed to ETS in the $3^{\text {rd }}$ trimester. There is also a low rate of attempting to quit smoking among pregnant women (52.3\%). Furthermore, individual SEP, assessed by educational level, was found to be predictive of smoking, smoking cessation and ETS exposure, which shows that further efforts on preventive and interventional measures to reduce smoking among the less educated are still of need. Therefore, public policies must continue to consider the reduction in inequalities in smoking among the most vulnerable social groups to, also, prevent future health inequalities.

\section{Data availability}

The datasets analyzed during the current study are available from the corresponding author on reasonable request.

Received: 6 May 2020; Accepted: 24 August 2020

Published online: 24 September 2020

\section{References}

1. WHO (World Health Organization). WHO European tobacco use. Trends report 2019. Copenhagen Denmark: World Health Organization. (2019)

2. Institute for Health Metrics and Evaluation (IHME). GBD compare Seattle, WA: IHME, University of Washington (2017). https ://vizhub.healthdata.org/gbd-compare/.

3. WHO (World Health Organization). WHO global report on trends in prevalence of tobacco use 2000-2025. Copenhagen Denmark: World Health Organization. (2019).

4. Leite, A., Machado, A., Pinto, S. Dias, C. M. Caraterísticas sociodemográficas dos fumadores diários em Portugal Continental: análise comparativa dos Inquéritos Nacionais de Saúde (1987, 1995/1996, 1998/1999, 2005/2006 e 2014). Ministério da Saúde. Instituto Nacional de Saúde Doutor Ricardo Jorge, IP-76 p il. (2017) (in Portuguese).

5. Cao, S., Yang, C., Gan, Y. \& Lu, Z. The health effects of passive smoking: an overview of systematic reviews based on observational epidemiological evidence. PLoS ONE 10(10), e0139907. https://doi.org/10.1371/journal.pone.0139907 (2015).

6. WHO (World Health Organization). Report on the global tobacco epidemic. the MPOWER package (World Health Organization, Geneva, 2008).

7. Secretan, B. et al. A review of human carcinogens-part E: tobacco, areca nut, alcohol, coal smoke, and salted fish. Lancet Oncol. 10(11), 1033-1034. https://doi.org/10.1016/s1470-2045(09)70326-2 (2009).

8. Inquérito Nacional de Saúde com Exame Físico (INSEF). Determinantes de saúde. Instituto Nacional de Saúde (2015). https:// repositorio.insa.pt//handle/10400.18/4795 (in Portuguese). 
9. Filippidis, T. F. et al. Relationship of secondhand smoke exposure with sociodemographic factors and smoke-free legislation in the European Union. Eur. J. Public Health 26(2), 344-349. https://doi.org/10.1093/eurpub/ckv204 (2015).

10. Lei 37/2017 Aprova normas para a proteção dos cidadãos da exposição involuntária ao fumo do tabaco e medidas de redução da procura relacionadas com a dependência e a cessação do seu consumo (2017) (Approves the regulations regarding the protection of citizens from involuntary exposure to tobacco smoke and measures to reduce demand associated with dependency and abandonment of tobacco use). data.dre.pt/eli/lei/37/2007/08/14/p/dre/pt/html (in Portuguese).

11. de Wolff, M. G. et al. Prevalence and predictors of maternal smoking prior to and during pregnancy in a regional Danish population: a cross-sectional study. Reprod. Health. 16, 82. https://doi.org/10.1186/s12978-019-0740-7 (2019).

12. Rumrich, I. K. et al. Smoking during pregnancy in Finland-trends in the MATEX cohort. Scand. J. Public. Health. 47(8), 890-898. https://doi.org/10.1177/1403494818804417 (2018).

13. Smedberg, J., Lupattelli, A., Mårdby, A. \& Nordeng, H. Characteristics of women who continue smoking during pregnancy: a cross-sectional study of pregnant women and new mothers in 15 European countries. BMC Pregnancy Childbirth 14, 213. https:// doi.org/10.1186/1471-2393-14-213 (2014).

14. Grøtvedt, L., Kvalvik, L. G., Grøholt, E. K., Akerkar, R. \& Egeland, G. M. Development of social and demographic differences in maternal smoking between 1999 and 2014 in Norway. Nicotine Tob. Res. 19(5), 539-546. https://doi.org/10.1093/ntr/ntw313 (2017).

15. Erlingsdottir, A., Sigurdsson, E. L., Jonsson, J. S., Kristjsandottir, H. \& Sigurdsson, J. A. Smoking during pregnancy: childbirth and health study in primary care in Iceland. Scand. J. Prim. Health Care. 32(1), 11-16. https://doi.org/10.3109/02813432.869409 (2014).

16. Alves, E., Azevedo, A., Correia, S. \& Barros, H. Long-term maintenance of smoking cessation in pregnancy: an analysis of the birth cohort generation XXI. Nicotine Tob. Res. 15(9), 1598-1607. https://doi.org/10.1093/ntr/ntt026 (2013).

17. Teixeira, C. et al. Tobacco use during pregnancy among native and migrant women in Portugal. Results from the Bambino study. Revue d'Épidémiologie et de Santé Publique 66(S5), S358-S359 (2018).

18. Abdulrahim, S. \& Jawad, M. Socioeconomic differences in smoking in Jordan, Lebanon, Syria, and Palestine: a cross-sectional analysis of national surveys. PLoS ONE 13(1), e0189829. https://doi.org/10.1371/journal.pone.0189829 (2018).

19. Casetta, B. et al. Association between cigarette smoking prevalence and income level: a systematic review and meta-analysis. Nicotine Tob. Res. 19(12), 1401-1407. https://doi.org/10.1093/ntr/ntw266 (2017).

20. Nicolaou, S. A., Heraclides, A., Markides, K. S. \& Charalambous, A. Prevalence and social determinants of smoking in the adult Greek Cypriot population. Hippokratia. 20(4), 284-291 (2016).

21. Cai, L. et al. Patterns and socioeconomic influences of tobacco exposure in tobacco cultivating rural areas of Yunnan province, China. BMC Public Health 12(1), 842. https://doi.org/10.1186/1471-2458-12-842 (2012).

22. Kondracki, A. J. Prevalence and patterns of cigarette smoking before and during early and late pregnancy according to maternal characteristics: the first national data based on the 2003 birth certificate revision, United States, 2016. Reprod. Health. 16(1), 142. https://doi.org/10.1186/s12978-019-0807-5 (2019).

23. Scheffers-van Schayck, T. S., Tuithof, M., Otten, R., Engels, R. \& Kleinjan, M. Smoking behavior of women before, during, and after pregnancy: indicators of smoking, quitting, and relapse. Eur. Addict. Res. 25(3), 132-144. https://doi.org/10.1159/000498988 (2019).

24. Miguez, M. C., Pereira, B. \& Figueiredo, B. Tobacco consumption and spontaneous quitting at the first trimester of pregnancy. Addict. Behav. 64, 111-117. https://doi.org/10.1016/j.addbeh.2016.08.034 (2017).

25. Xu, X. et al. Smoking in pregnancy: a cross-sectional study in China. Tob. Induc. Dis. 15, 35. https://doi.org/10.1186/s12971-0170140-0 (2017).

26. Rocheleau, C. M. et al. Factors associated with employment status before and during pregnancy: implications for studies of pregnancy outcomes. Am. J. Ind. Med. 60(4), 329-341. https://doi.org/10.1002/ajim.22700 (2017).

27. Duncan, C., Jones, K. \& Moon, G. Smoking and deprivation: are there neighbourhood effects?. Soc. Sci. Med. 48(4), 497-505. https ://doi.org/10.1016/s0277-9536(98)00360-8 (1999).

28. Baxter, J. \& Matthew, T. Measuring the socio-economic status of women across the life course. Family Matters. 95, 62 (2014).

29. Tran, D. T., Alys, H. \& Louisa, R. J. Data cleaning and management protocols for linked perinatal research data: a good practice example from the smoking MUMS (maternal use of medications and safety) study. BMC Med. Res. Methodol. 17, 97. https://doi. org/10.1186/s12874-017-0385-6 (2017).

30. Ribeiro, A. I., Olhero, A., Teixeira, H., Magalhães, A. \& Pina, M. F. Tools for address georeferencing-limitations and opportunities every public health professional should be aware of. PLoS ONE 9(12), e114130. https://doi.org/10.1371/journal.pone.01141 30 (2014).

31. INE (Instituto Nacional de Estatística) https://www.ine.pt/xportal/xmain?xpid=INE\&xpgid=ine_cont_inst\&INST=6251013\&xlang =pt. accessed 17 April 2020 (in Portuguese).

32. Park, E.-J., Kim, H., Kawachi, I., Kim, I.-L. \& Cho, S.-I. Area deprivation, individual socioeconomic position and smoking among women in South Korea. Tob Control. 19(5), 383-390. https://doi.org/10.1136/tc.2009.032763 (2010).

33. Ribeiro, A. I., Launay, L., Guillaume, E., Launoy, G. \& Barros, H. The Portuguese version of the European deprivation index: development and association with all-cause mortality. PLoS ONE 13(2), e0208320. https://doi.org/10.1371/journal.pone.0208320 (2018).

34. Global Tobacco Surveillance System. Global Adult Tobacco Survey (GATS): Indicator Guidelines: Definition and Syntax. (2009).

35. Blatt, K., Moore, E., Chen, A., Hook, J. V. \& DeFranco, E. A. Association of reported trimester-specific smoking cessation and fetal growth restriction. Obstet Gynecol. 125(6), 1452-1459. https://doi.org/10.1097/AOG.0000000000000679 (2015).

36. Mund, M., Louwen, F., Klingelhoefer, D. \& Gerber, A. Smoking and pregnancy-a review on the first major environmental risk factor of the unborn. Int. J. Environ. Res. Public. Health. 10(12), 6485-6499. https://doi.org/10.3390/ijerph10126485 (2013).

37. Decreto-Lei no. 73/2010-Código dos Impostos Especiais de Consumo (2010) (Approves special consumption tax codes). https ://data.dre.pt/eli/dec-lei/73/2010/06/21/p/dre/pt/html (in Portuguese).

38. Taype-Rondan, A. et al. Smoking and heavy drinking patterns in rural, urban and rural-to-urban migrants: the PERU MIGRANT Study. BMC Public Health 17(1), 165. https://doi.org/10.1186/s12889-017-4080-7 (2017).

39. Milcarz, M., Polanska, K., Bak-Romanisyzn, L. \& Kaleta, D. Tobacco health risk awareness among socially disadvantaged people-a crucial tool for smoking cessation. Int. J. Environ. Res. Public Health 15(10), 2244. https://doi.org/10.3390/ijerph15102244 (2018).

40. Boucher, J. \& Konkle, A. T. M. Understanding inequalities of maternal smoking-bridging the gap with adapted intervention strategies. Int. J. Environ. Res. Public. Health 13(3), 282. https://doi.org/10.3390/ijerph13030282 (2016).

41. Bista, B. et al. Socio-demographic predictors of tobacco use among women of Nepal: evidence from non-communicable disease risk factors STEPS Survey Nepal 2013. J. Nepal Health Res. Counc. 13(29), 14-19 (2015).

42. Hiscock, R., Judge, K. \& Bauld, L. Social inequalities in quitting smoking: what factors mediate the relationship between socioeconomic position and smoking cessation?. J. Public Health. 33(1), 39-47. https://doi.org/10.1093/pubmed/fdq097 (2011).

43. Kia, F., Tosun, N., Carlson, S. \& Allen, S. Examining characteristics associated with quitting smoking during pregnancy and relapse postpartum. Addict Behav. 78, 114-119. https://doi.org/10.1016/j.addbeh.2017.11.011 (2018).

44. Galobardes, B., Shaw, M., Lawlor, D. A. \& Lynch, J. W. Indicators of socioeconomic position (part 1). J. Epidemiol. Community Health. 60(1), 7-12. https://doi.org/10.1136/jech.2004.023531 (2006).

45. Flemming, K., Graham, H., Heirs, M., Fox, D. \& Sowden, A. Smoking in pregnancy: a systematic review of qualitative research of women who commence pregnancy as smokers. J. Adv. Nurs. 69(5), 1023-1036. https://doi.org/10.1111/jan.12066 (2013). 
46. Crozier, S. R. et al. Do women change their health behaviours in pregnancy? Findings from the Southampton women's survey. Paediatr. Perinat. Epidemiol. 23(5), 446-453. https://doi.org/10.1111/j.1365-3016.2009.01036.x (2009).

47. McBride, C. M., Emmons, K. M. \& Lipkus, I. M. Understanding the potential of teachable moments: the case of smoking cessation. Health Educ. Res. 18(2), 156-170. https://doi.org/10.1093/her/18.2.156 (2003).

48. Yang, L., Tong, E. K., Mao, Z. \& Hu, T. Exposure to secondhand smoke and associated factors among non-smoking pregnant women with smoking husbands in Sichuan province, China. Acta Obstet. Gynecol. Scand. 89(4), 549-557. https://doi.org/10.3109/00016 341003713851 (2010).

49. Alghamdi, A. S. et al. Socioeconomic determinants of exposure to secondhand smoke among pregnant women. Int. J. Women's Health Reprod. Sci. 4(2), 59-63. https://doi.org/10.15296/ijwhr.2016.14 (2016).

50. Bernstein, S. L., Rosner, J. \& Toll, B. Concordance between timeline follow-back and single-question assessment of self-reported smoking in a clinical trial. Subst. Abus. 37(3), 398-401. https://doi.org/10.1080/08897077.2016.1154494 (2016).

51. Mattsson, K. et al. Cotinine validation of self-reported smoking during pregnancy in the Swedish medical birth register. Nicotine Tob. Res. 18(1), 79-83. https://doi.org/10.1093/ntr/ntv087 (2016).

52. Oakes, J. M. \& Rossi, P. H. The measurement of SES in health research: current practice and steps toward a new approach. Soc Sci Med. 56(4), 769-784. https://doi.org/10.1016/S0277-9536(02)00073-4 (2003).

\title{
Acknowledgments
}

This work was supported by FCT and FAPESP (FAPESP/19914/2014) and by FEDER through the Operational Programme Competitiveness and Internationalization and national funding from the Foundation for Science and Technology_FCT (Portuguese Ministry of Science, Technology and Higher Education) under the Unidade de Investigação em Epidemiologia-Instituto de Saúde Pública da Universidade do Porto (EPIUnit) (POCI-01-0145FEDER-006862; Ref. UID/DTP/04750/2019). Joana Madureira, Carla Costa and Ana Inês Silva are supported by FCT (SFRH/BPD/115112/2016, SFRH/BPD/96196/2013 and SFRH/BD/145101/2019 grants, respectively).

\section{Author contributions}

J.M. lead the conceptualization, formal analysis and wrote the main manuscript text. A.C. lead the data curation and wrote the main manuscript. A.I.S., A.T.R. and F.E. contributed for data collected and reviewed the manuscript. AIR supported the formal analysis and reviewed/edited the manuscript. J.P.T. reviewed the manuscript and provided all the resources. C.C. coordinated the project, assured the funding, co-lead the conceptualization, and reviewed/edited the manuscript.

\section{Competing interests}

The authors declare no competing interests.

\section{Additional information}

Supplementary information is available for this paper at https://doi.org/10.1038/s41598-020-72298-8.

Correspondence and requests for materials should be addressed to J.P.T.

Reprints and permissions information is available at www.nature.com/reprints.

Publisher's note Springer Nature remains neutral with regard to jurisdictional claims in published maps and institutional affiliations.

\begin{abstract}
Open Access This article is licensed under a Creative Commons Attribution 4.0 International License, which permits use, sharing, adaptation, distribution and reproduction in any medium or format, as long as you give appropriate credit to the original author(s) and the source, provide a link to the Creative Commons licence, and indicate if changes were made. The images or other third party material in this article are included in the article's Creative Commons licence, unless indicated otherwise in a credit line to the material. If material is not included in the article's Creative Commons licence and your intended use is not permitted by statutory regulation or exceeds the permitted use, you will need to obtain permission directly from the copyright holder. To view a copy of this licence, visit http://creativecommons.org/licenses/by/4.0/.
\end{abstract}

(C) The Author(s) 2020 